\title{
On the existence of solutions for a
}

\section{fractional finite difference inclusion via three points boundary conditions}

\section{Dumitru Baleanu ${ }^{1,2^{*}}$, Shahram Rezapour ${ }^{3}$ and Saeid Salehi ${ }^{3}$}

*Correspondence:

dumitru@cankaya.edu.tr

1 Department of Mathematics,

Cankaya University, Ogretmenler

Cad. 14, Balgat, Ankara, 06530,

Turkey

${ }^{2}$ Institute of Space Sciences,

Magurele, Bucharest, Romania

Full list of author information is

available at the end of the article

\begin{abstract}
In this paper, we discussed the existence of solutions for the fractional finite difference inclusion $\Delta^{v} x(t) \in F\left(t, x(t), \Delta x(t), \Delta^{2} x(t)\right)$ via the boundary value conditions $\xi x(v-3)+\beta \Delta x(v-3)=0, x(\eta)=0$, and $\gamma x(b+v)+\delta \Delta x(b+v)=0$, where $\eta \in \mathbb{N}_{v-2}^{b+\nu-1}$, $2<v<3$, and $F: \mathbb{N}_{\nu-3}^{b+\nu+1} \times \mathbb{R} \times \mathbb{R} \times \mathbb{R} \rightarrow 2^{\mathbb{R}}$ is a compact valued multifunction.
\end{abstract}

Keywords: fixed point; fractional finite difference inclusion; three points boundary conditions

\section{Introduction}

There are many works concerned with the existence of solutions for some fractional finite difference equations from different views by using the fixed point theory techniques (see for example, [1-7]). The readers can find more details as regards elementary notions and definitions of fractional finite difference equations in [8-15]. Also, much attention was devoted to the fractional differential inclusions (see for example, $[9,10,16-24]$ ). To the best of our knowledge, there is no published research work about fractional finite difference inclusions.

In 2011, Goodrich [25] investigated the general discrete fractional boundary problem, namely

$$
\left\{\begin{array}{l}
-\Delta^{v} y(t)=f(t+v-1, y(t+v-1)), \\
\alpha y(v-2)-\beta \Delta y(v-2)=0 \\
\gamma y(v+b)-\delta \Delta y(v+b)=0
\end{array}\right.
$$

where $t \in[0, b]_{\mathbb{N}_{0}}, v \in(1,2]$, and $\alpha \gamma+\alpha \delta+\beta \gamma \neq 0$ with $\alpha, \beta, \gamma, \delta \geq 0$. In this paper, with this thought and motivation in our minds, we investigate the existence of solution for the fractional finite difference inclusion

$$
\left\{\begin{array}{l}
\Delta^{v} x(t) \in F\left(t, x(t), \Delta x(t), \Delta^{2} x(t)\right), \\
\xi x(v-3)+\beta \Delta x(v-3)=0, \\
x(\eta)=0, \\
\gamma x(b+v)+\delta \Delta x(b+v)=0,
\end{array}\right.
$$

(c) 2015 Baleanu et al. This article is distributed under the terms of the Creative Commons Attribution 4.0 International License (http://creativecommons.org/licenses/by/4.0/), which permits unrestricted use, distribution, and reproduction in any medium, provided you give appropriate credit to the original author(s) and the source, provide a link to the Creative Commons license, and indicate if changes were made. 
where $\eta \in \mathbb{N}_{\nu-2}^{b+\nu-1}, 2<\nu<3$ and $F: \mathbb{N}_{\nu-3}^{b+\nu+1} \times \mathbb{R} \times \mathbb{R} \times \mathbb{R} \rightarrow 2^{\mathbb{R}}$ is a compact valued multifunction.

\section{Preliminaries}

As is well known, the Gamma function has some properties as $\Gamma(z+1)=z \Gamma(z)$ and $\Gamma(n)=$ $(n-1)$ ! for all $n \in \mathbb{N}$. Define

$$
t^{\underline{v}}=\frac{\Gamma(t+1)}{\Gamma(t+1-v)}
$$

for all $t, v \in \mathbb{R}$ whenever the right-hand side is defined. If $t+1-v$ is a pole of the gamma function and $t+1$ is not a pole, then we define $t^{\underline{v}}=0$. One can verify that $\nu^{\underline{v}}=v \underline{v-1}=$ $\Gamma(v+1)$ and $t^{\underline{\nu+1}}=(t-v) t^{\underline{\nu}}$. We use the notations $\mathbb{N}_{a}=\{a, a+1, a+2, \ldots\}$ for all $a \in \mathbb{R}$ and $\mathbb{N}_{a}^{b}=\{a, a+1, a+2, \ldots, b\}$ for all real numbers $a$ and $b$ whenever $b-a$ is a natural number.

Let $v>0$ be such that $m-1<v \leq m$ for some natural number $m$. Then the $v$ th fractional sum of $f$ based at $a$ is defined by

$$
\Delta_{a}^{-v} f(t)=\frac{1}{\Gamma(v)} \sum_{k=a}^{t-v}(t-\sigma(k))^{\frac{v-1}{} f(k)}
$$

for all $t \in \mathbb{N}_{a+\nu}$. Similarly, we define

$$
\Delta_{a}^{v} f(t)=\frac{1}{\Gamma(-v)} \sum_{k=a}^{t+v}(t-\sigma(k))^{-v-1} f(k)
$$

for all $t \in \mathbb{N}_{a+m-\nu}$.

Lemma 2.1 [1] Let $h: \mathbb{N}_{v-3}^{b+v+1} \rightarrow \mathbb{R}$ be a mapping and $2<v \leq 3$. The general solution of the equation $\Delta_{v-3}^{v} x(t)=h(t)$ is given by

$$
x(t)=\sum_{i=1}^{3} c_{i} t^{\frac{\nu-i}{}}+\frac{1}{\Gamma(v)} \sum_{s=0}^{t-v}(t-\sigma(s))^{\frac{v-1}{h}} h(s),
$$

where $c_{1}, c_{2}, c_{3}$ are arbitrary constants.

Since $\Delta t^{\underline{\mu}}=\mu t^{\mu-1}$, we have

$$
\Delta x(t)=\sum_{i=1}^{3} c_{i}(\nu-i) t^{\nu-i-1}+\frac{1}{\Gamma(\nu-1)} \sum_{s=0}^{t-v+1}(t-\sigma(s))^{\frac{\nu-2}{h}} h(s)
$$

for more information see [12].

Let $(X, d)$ be a metric space. Denote by $2^{X}, C B(X)$, and $P_{\mathrm{cp}}(X)$ the class of all nonempty subsets, the class of all closed and bounded subsets, and the class of all compact subsets of $X$, respectively. A mapping $Q: X \rightarrow 2^{X}$ is called a multifunction on $X$ and $u \in X$ is called a fixed point of $Q$ whenever $u \in Q u$.

Consider the Hausdorff metric $H_{d}: 2^{X} \times 2^{X} \rightarrow[0, \infty)$ by

$$
H_{d}(A, B)=\max \left\{\sup _{a \in A} d(a, B), \sup _{b \in B} d(A, b)\right\}
$$


where $d(A, b)=\inf _{a \in A} d(a, b)$. Let $(X, d)$ be a metric space, $\alpha: X \times X \rightarrow[0, \infty)$ a map, and $T: X \rightarrow 2^{X}$ a multifunction.

We say that $X$ obeys the condition $\left(C_{\alpha}\right)$ whenever for each sequence $\left\{x_{n}\right\}$ in $X$ with $\alpha\left(x_{n}, x_{n+1}\right) \geq 1$ for all $n$ and $x_{n} \rightarrow x$, there exists a subsequence $\left\{x_{n_{k}}\right\}$ of $\left\{x_{n}\right\}$ such that $\alpha\left(x_{n_{k}}, x\right) \geq 1$ for all $k$. The map $T$ is said to be $\alpha$-admissible whenever for each $x \in X$ and $y \in T x$ with $\alpha(x, y) \geq 1$, we have $\alpha(y, z) \geq 1$ for all $z \in T y$ [26]. Suppose that $\Psi$ is the family of nondecreasing functions $\psi:[0, \infty) \rightarrow[0, \infty)$ such that $\sum_{n=1}^{\infty} \psi^{n}(t)<\infty$ for all $t>0$ (for more on this please see [26]).

By using the following fixed point result, we review the existence of solutions for the fractional finite difference inclusion

$$
\Delta_{v-3}^{v} x(t) \in F\left(t, x(t), \Delta x(t), \Delta^{2} x(t)\right)
$$

via the boundary conditions $\xi x(v-3)+\beta \Delta x(v-3)=0, \gamma x(b+v)+\delta \Delta x(b+v)=0$, and $x(\eta)=0$, where $\eta \in \mathbb{N}_{\nu-2}^{b+\nu-1}, 2<\nu<3$, and $F: \mathbb{N}_{\nu-3}^{b+\nu} \times \mathbb{R} \times \mathbb{R} \times \mathbb{R} \rightarrow 2^{\mathbb{R}}$ is a compact valued multifunction.

Lemma 2.2 [26] Let $(X, d)$ be a complete metric space, $\psi \in \Psi$ a strictly increasing map, $\alpha: X \times X \rightarrow[0, \infty)$ a map and $T: X \rightarrow C B(X)$ an $\alpha$-admissible multifunction such that $\alpha(x, y) H(T x, T y) \leq \psi(d(x, y))$ for all $x, y \in X$ and there exist $x_{0} \in X$ and $x_{1} \in T x_{0}$ with $\alpha\left(x_{0}, x_{1}\right) \geq 1$. If $X$ obeys the condition $\left(C_{\alpha}\right)$, then $T$ has a fixed point.

\section{Main result}

In this section, we consider the fractional finite difference inclusion

$$
\Delta_{v-3}^{v} x(t) \in F\left(t, x(t), \Delta x(t), \Delta^{2} x(t)\right)
$$

via the boundary value conditions $\xi x(v-3)+\beta \Delta x(v-3)=0, \gamma x(b+v)+\delta \Delta x(b+v)=0$, and $x(\eta)=0$, where $\xi, \beta, \gamma, \delta$ are non-zero numbers, $\eta \in \mathbb{N}_{v-2}^{b+\nu-1}, 2<\nu<3, x: \mathbb{N}_{\nu-3}^{b+v+1} \rightarrow \mathbb{R}$ and $F: \mathbb{N}_{\nu-3}^{b+v+1} \times \mathbb{R} \times \mathbb{R} \times \mathbb{R} \rightarrow 2^{\mathbb{R}}$ is a compact valued multifunction.

Lemma 3.1 Let $y: \mathbb{N}_{0}^{b+1} \rightarrow \mathbb{R}$ and $2<v<3$. Then $x_{0}$ is a solution for the fractional finite difference equation $\Delta_{v-3}^{v} x(t)=y(t)$ via the boundary conditions $\xi x(v-3)+\beta \Delta x(v-3)=0$, $x(\eta)=0$, and $\gamma x(b+v)+\delta \Delta x(b+v)=0$ if and only if $x_{0}$ is a solution of the fractional sum equation $x(t)=\sum_{s=0}^{b+1} G(t, s, \eta) y(s)$, where

$$
\begin{aligned}
& G(t, s, \eta)=\left[\frac{[\gamma+\delta(v-1)][(\eta+2-v)(\eta+3-v)] t \underline{\underline{v-3}}-\theta[\gamma+\delta(v-1)] t \underline{\underline{\nu-1}}}{\theta \beta_{0} \mu \Gamma(v)(b+v) \underline{v-4}}\right. \\
& \left.-\frac{[\xi-\beta(v-3)][\gamma+\delta(v-1)][(\eta+2-v)(\eta+3-v)] t^{\frac{v-2}{2}}}{\beta(v-2) \theta \beta_{0} \mu \Gamma(v)(b+v) \underline{v-4}}\right]
\end{aligned}
$$

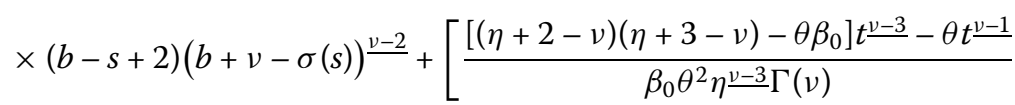

$$
\begin{aligned}
& \left.+\frac{[-\xi+\beta(v-3)]\left[(\eta+2-v)(\eta+3-v)-\theta \beta_{0}\right] t^{\nu-2}}{\beta(v-2) \theta^{2} \beta_{0} \eta \underline{\underline{v-3}} \Gamma(v)}\right](\eta-\sigma(s))^{\frac{v-1}{\underline{y}}} \\
& +\frac{(t-\sigma(s)) \frac{v-1}{\Gamma(v)}}{,}
\end{aligned}
$$


whenever $0 \leq s \leq t-v \leq b+1$ and $0 \leq s \leq \eta-v \leq b+1$,

$$
\begin{aligned}
& G(t, s, \eta)=\left[\frac{[\gamma+\delta(v-1)][(\eta+2-v)(\eta+3-v)] t \frac{v-3}{v-4}-\theta[\gamma+\delta(v-1)] t^{v-1}}{\theta \beta_{0} \mu \Gamma(v)(b+v) \underline{v-4}}\right. \\
& \left.-\frac{[\xi-\beta(v-3)][\gamma+\delta(v-1)][(\eta+2-v)(\eta+3-v)] t \underline{v-2}}{\beta(v-2) \theta \beta_{0} \mu \Gamma(v)(b+v) \underline{v-4}}\right] \\
& \times(b-s+2)(b+v-\sigma(s))^{\frac{v-2}{}}+\left[\frac{\left[(\eta+2-v)(\eta+3-v)-\theta \beta_{0}\right] t \frac{v-3}{-\theta}-\theta t \frac{v-1}{2}}{\beta_{0} \theta^{2} \eta^{\frac{\nu-3}{2}} \Gamma(v)}\right. \\
& \left.+\frac{[-\xi+\beta(v-3)]\left[(\eta+2-v)(\eta+3-v)-\theta \beta_{0}\right] t \frac{v-2}{2}}{\beta(v-2) \theta^{2} \beta_{0} \eta \frac{v-3}{} \Gamma(v)}\right](\eta-\sigma(s))^{\frac{v-1}{}},
\end{aligned}
$$

whenever $0 \leq t-v<s \leq \eta-v \leq b+1$,

$$
\begin{aligned}
& G(t, s, \eta)=\left[\frac{[\gamma+\delta(v-1)][(\eta+2-v)(\eta+3-v)] t \frac{v-3}{n}-\theta[\gamma+\delta(v-1)] t^{\frac{\nu-1}{2}}}{\theta \beta_{0} \mu \Gamma(\nu)(b+v) \underline{v-4}}\right. \\
& \left.-\frac{[\xi-\beta(v-3)][\gamma+\delta(v-1)][(\eta+2-v)(\eta+3-v)] t \underline{v-2}}{\beta(v-2) \theta \beta_{0} \mu \Gamma(v)(b+v) \underline{v-4}}\right] \\
& \times(b-s+2)(b+v-\sigma(s))^{\frac{v-2}{}}+\frac{(t-\sigma(s))^{\frac{v-1}{2}}}{\Gamma(v)},
\end{aligned}
$$

whenever $0 \leq \eta-v<s \leq t-v \leq b+1$ and

$$
\begin{aligned}
G(t, s, \eta)= & {\left[\frac{[\gamma+\delta(v-1)][(\eta+2-v)(\eta+3-v)] t \frac{v-3}{v}-\theta[\gamma+\delta(v-1)] t \frac{v-1}{\underline{v}}}{\theta \beta_{0} \mu \Gamma(\nu)(b+v)}\right.} \\
& \left.-\frac{[\xi-\beta(v-3)][\gamma+\delta(v-1)][(\eta+2-v)(\eta+3-v)] t \frac{v-2}{2}}{\beta(v-2) \theta \beta_{0} \mu \Gamma(v)(b+v) \underline{v-4}}\right] \\
& \times(b-s+2)(b+v-\sigma(s))^{\frac{\nu-2}{2}}
\end{aligned}
$$

whenever $0 \leq t-v<s \leq b+1$ and $0 \leq \eta-v<s \leq b+1$. Here,

$$
\begin{aligned}
\theta= & \frac{\eta \beta v-\eta \xi-3 \eta \beta-2 \xi+\xi v-\beta v^{2}+6 \beta v-8 \beta}{\beta(v-2)}, \\
\mu= & \frac{b \xi \delta v-2 b \delta \xi+\gamma \xi b^{2}+3 b \gamma \xi+\beta b v^{2} \delta+\delta b^{2} \beta v+\beta b \delta v-6 \beta \delta b+3 \beta \delta b^{2}+4 \xi \delta v}{\beta(v-2)} \\
& +\frac{-8 \delta \xi+4 \gamma \xi b+12 \gamma \xi+4 \beta v^{2} \delta+7 \gamma \beta v b+12 \gamma \beta v+4 \beta \delta v-24 \beta \delta+21 \beta \gamma b+36 \beta \gamma}{\beta(v-2)}
\end{aligned}
$$

and

$$
\beta_{0}=\frac{\theta[\delta(v-1)+\gamma(b+2)](b+3)(b+4)+\mu(\eta+2-v)(\eta+3-v)}{\theta \mu} .
$$

Proof Let $x_{0}$ be a solution for the equation $\Delta_{v-3}^{v} x(t)=y(t)$ via the boundary conditions $\xi x(v-3)+\beta \Delta x(v-3)=0, x(\eta)=0$, and $\gamma x(b+v)+\delta \Delta x(b+v)=0$. Then by using (2) and Lemma 2.1, we get

$$
x_{0}(t)=c_{1} t^{\frac{\nu-1}{2}}+c_{2} t^{\frac{\nu-2}{2}}+c_{3} t^{\frac{\nu-3}{}}+\frac{1}{\Gamma(\nu)} \sum_{s=0}^{t-v}(t-\sigma(s))^{\frac{\nu-1}{v}} y(s)
$$


and

$$
\begin{aligned}
\Delta x_{0}(t)= & c_{1}(v-1) t \frac{v-2}{2}+c_{2}(v-2) t^{\frac{\nu-3}{}}+c_{3}(v-3) t^{\frac{\nu-4}{}} \\
& +\frac{1}{\Gamma(v-1)} \sum_{s=0}^{t-v+1}(t-\sigma(s))^{\frac{v-2}{y}} y(s)
\end{aligned}
$$

where $c_{1}, c_{2}, c_{3} \in \mathbb{R}$ are arbitrary constants. Now, by using the boundary condition

$$
\xi x(v-3)+\beta \Delta x(v-3)=0,
$$

we get $\xi c_{3}+\beta\left[c_{2}(v-2)+c_{3}(v-3)\right]=0$. Also, by using the condition $x(\eta)=0$ we obtain

$$
\begin{gathered}
c_{3}=-(\eta+2-v)(\eta+3-v) c_{1}-(\eta+2-v) c_{2} \\
-\frac{1}{\eta \frac{v-3}{\Gamma} \Gamma(\nu)} \sum_{s=0}^{\eta-v}(\eta-\sigma(s))^{\frac{\nu-1}{y}} y(s) .
\end{gathered}
$$

Moreover, by using the boundary condition $\gamma x(b+v)+\delta \Delta x(b+v)=0$, we get

$$
\begin{aligned}
c_{1}[\delta(v-1)+\gamma(b+2)](b+v)^{\frac{v-2}{2}}+c_{2}[\delta(v-2)+\gamma(b+3)](b+v)^{\frac{v-3}{u}} & \\
& +c_{3}[\delta(v-3)+\gamma(b+4)](b+v)^{\frac{v-4}{}} \\
= & -\frac{\delta}{\Gamma(v-1)} \sum_{s=0}^{b+1}(b+v-\sigma(s))^{\frac{v-2}{}} y(s)-\frac{\gamma}{\Gamma(v)} \sum_{s=0}^{b}(b+v-\sigma(s))^{\frac{v-1}{v}} y(s) .
\end{aligned}
$$

Thus, by using a simple calculation, we get

$$
\begin{aligned}
& c_{1}=-\frac{1}{\beta_{0} \theta \eta \frac{\nu-3}{-} \Gamma(\nu)} \sum_{s=0}^{\eta-v}(\eta-\sigma(s))^{\frac{\nu-1}{\gamma}} y(s) \\
& -\frac{\gamma+\delta(v-1)}{\beta_{0} \mu \Gamma(v)(b+v) \frac{v-4}{b+1}} \sum_{s=0}^{b}(b-s+2)(b+v-\sigma(s))^{\frac{v-2}{y}} y(s), \\
& c_{2}=\frac{[-\xi+\beta(v-3)]\left[(\eta+2-v)(\eta+3-v)-\theta \beta_{0}\right]}{\beta(v-2) \theta^{2} \beta_{0} \eta \frac{v-3}{-} \Gamma(\nu)} \sum_{s=0}^{\eta-v}(\eta-\sigma(s))^{\frac{v-1}{y}} y(s) \\
& -\frac{[\xi-\beta(v-3)][\gamma+\delta(v-1)][(\eta+2-v)(\eta+3-v)]}{\beta(v-2) \theta \beta_{0} \mu \Gamma(v)(b+v) \underline{v-4}} \\
& \times \sum_{s=0}^{b+1}(b-s+2)(b+v-\sigma(s))^{\frac{v-2}{y}} y(s)
\end{aligned}
$$

and

$$
\begin{aligned}
c_{3}= & \frac{(\eta+2-v)(\eta+3-v)-\theta \beta_{0}}{\theta^{2} \beta_{0} \eta \frac{v-3}{\eta} \Gamma(v)} \sum_{s=0}^{\eta-v}(\eta-\sigma(s))^{\frac{v-1}{y}} y(s) \\
& +\frac{[\gamma+\delta(v-1)][(\eta+2-v)(\eta+3-v)]}{\theta \beta_{0} \mu \Gamma(v)(b+v) \frac{v-4}{2}} \sum_{s=0}^{b+1}(b-s+2)(b+v-\sigma(s))^{\frac{\nu-2}{}} y(s) .
\end{aligned}
$$


Hence,

$$
\begin{aligned}
& x_{0}(t)=\left[\frac{[\gamma+\delta(v-1)][(\eta+2-v)(\eta+3-v)] t \underline{v-3}-\theta[\gamma+\delta(v-1)] t \frac{v-1}{}}{\theta \beta_{0} \mu \Gamma(\nu)(b+v) \underline{v-4}}\right. \\
& \left.-\frac{[\xi-\beta(v-3)][\gamma+\delta(v-1)][(\eta+2-v)(\eta+3-v)] t \underline{v-2}}{\beta(v-2) \theta \beta_{0} \mu \Gamma(v)(b+v) \underline{v-4}}\right] \\
& \times \sum_{s=0}^{b+1}(b-s+2)(b+v-\sigma(s))^{\frac{v-2}{y}} y(s) \\
& +\left[\frac{\left[(\eta+2-v)(\eta+3-v)-\theta \beta_{0}\right] t \frac{v-3}{-}-\theta t \frac{v-1}{\underline{v}}}{\beta_{0} \theta^{2} \eta \frac{v-3}{\Gamma} \Gamma(\nu)}\right. \\
& \left.+\frac{[-\xi+\beta(v-3)]\left[(\eta+2-v)(\eta+3-v)-\theta \beta_{0}\right] t \frac{v-2}{\eta-v}}{\beta(v-2) \theta^{2} \beta_{0} \eta \frac{v-3}{n} \Gamma(v)}\right] \sum_{s=0}^{\eta-v}(\eta-\sigma(s))^{\frac{v-1}{y}} y(s) \\
& +\sum_{s=0}^{t-v} \frac{(t-\sigma(s))^{\frac{\nu-1}{}}}{\Gamma(v)} y(s)=\sum_{s=0}^{b+1} G(s, t, \eta) y(s) .
\end{aligned}
$$

Now, let $x_{0}$ be a solution for the equation $x(t)=\sum_{s=0}^{b+1} G(s, t, \eta) y(s)$. Then we have

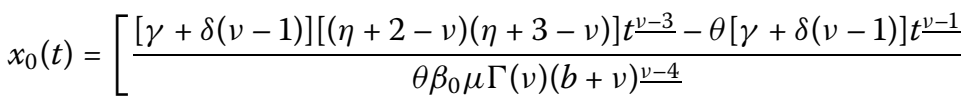

$$
\begin{aligned}
& \left.-\frac{[\xi-\beta(v-3)][\gamma+\delta(v-1)][(\eta+2-v)(\eta+3-v)] t \underline{v-2}}{\beta(v-2) \theta \beta_{0} \mu \Gamma(v)(b+v) \underline{v-4}}\right] \\
& \times \sum_{s=0}^{b+1}(b-s+2)(b+v-\sigma(s))^{\frac{v-2}{y}} y(s) \\
& +\left[\frac{\left[(\eta+2-v)(\eta+3-v)-\theta \beta_{0}\right] t \frac{v-3}{-\theta}-\theta t \underline{v-1}}{\beta_{0} \theta^{2} \eta \frac{v-3}{} \Gamma(\nu)}\right. \\
& \left.+\frac{[-\xi+\beta(v-3)]\left[(\eta+2-v)(\eta+3-v)-\theta \beta_{0}\right] t \frac{v-2}{2}}{\beta(v-2) \theta^{2} \beta_{0} \eta \frac{v-3}{-} \Gamma(v)}\right] \\
& \times \sum_{s=0}^{\eta-v}(\eta-\sigma(s))^{\frac{v-1}{y}} y(s)+\sum_{s=0}^{t-v} \frac{(t-\sigma(s))^{\frac{\nu-1}{2}}}{\Gamma(v)} y(s) .
\end{aligned}
$$

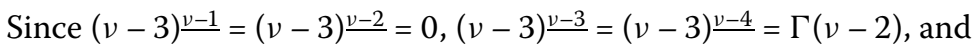

$$
\sum_{s=0}^{-3}(v-3-\sigma(s))^{\frac{v-1}{y}} y(s)=\sum_{s=0}^{-2}(v-3-\sigma(s))^{\frac{v-2}{2}} y(s)=0,
$$

we get $\xi x_{0}(v-3)+\beta \Delta x_{0}(v-3)=0$. A simple calculation shows us $\gamma x_{0}(b+v)+\delta \Delta x_{0}(b+v)=$ 0 and $x_{0}(\eta)=0$. On the other hand,

$$
x_{0}(t)=c_{1} t^{\frac{\nu-1}{v}}+c_{2} t^{\frac{\nu-2}{2}}+c_{3} t^{\frac{\nu-3}{}}+\frac{1}{\Gamma(\nu)} \sum_{s=0}^{t-v}(t-\sigma(s))^{\frac{\nu-1}{}} y(s)
$$

is a solution for the equation $\Delta_{v-3}^{v} x(t)=y(t)$ and so $\Delta_{v-3}^{v} x_{0}(t)=y(t)$. 
A function $x: \mathbb{N}_{\nu-3}^{b+v+1} \rightarrow \mathbb{R}$ is a solution of the problem (3) whenever it satisfies the boundary conditions and there exists a function $y: \mathbb{N}_{0}^{b+1} \rightarrow \mathbb{R}$ such that

$$
y(t) \in F\left(t, x(t), \Delta x(t), \Delta^{2} x(t)\right)
$$

for all $t \in \mathbb{N}_{0}^{b+1}$ and

$$
\begin{aligned}
& x(t)=\left[\frac{[\gamma+\delta(\nu-1)][(\eta+2-v)(\eta+3-v)] t^{\nu-3}-\theta[\gamma+\delta(\nu-1)] t^{\nu-1}}{\theta \beta_{0} \mu \Gamma(v)(b+v) \underline{v-4}}\right. \\
& \left.-\frac{[\xi-\beta(v-3)][\gamma+\delta(v-1)][(\eta+2-v)(\eta+3-v)] t \frac{v-2}{\underline{v}}}{\beta(v-2) \theta \beta_{0} \mu \Gamma(v)(b+v) \underline{\underline{v}-4}}\right] \\
& \times \sum_{s=0}^{b+1}(b-s+2)(b+v-\sigma(s))^{\frac{v-2}{y}} y(s) \\
& +\left[\frac{\left[(\eta+2-v)(\eta+3-v)-\theta \beta_{0}\right] t^{\underline{\nu-3}}-\theta t^{\underline{\nu-1}}}{\beta_{0} \theta^{2} \eta^{\underline{\nu-3}} \Gamma(v)}\right. \\
& \left.+\frac{[-\xi+\beta(v-3)]\left[(\eta+2-v)(\eta+3-v)-\theta \beta_{0}\right] t \frac{v-2}{2}}{\beta(v-2) \theta^{2} \beta_{0} \eta \underline{v-3} \Gamma(v)}\right] \\
& \times \sum_{s=0}^{\eta-v}(\eta-\sigma(s))^{\frac{\nu-1}{v}} y(s)+\sum_{s=0}^{t-v} \frac{(t-\sigma(s))^{\frac{\nu-1}{}}}{\Gamma(v)} y(s) .
\end{aligned}
$$

Let $\mathcal{X}$ be the set of all functions $x: \mathbb{N}_{\nu-3}^{b+\nu+1} \rightarrow \mathbb{R}$ endowed with the norm

$$
\|x\|=\max _{t \in \mathbb{N}_{v-3}^{b+1}}|x(t)|+\max _{t \in \mathbb{N}_{v-3}^{b+v+1}}|\Delta x(t)|+\max _{t \in \mathbb{N}_{\nu-3}^{b+v+1}}\left|\Delta^{2} x(t)\right| .
$$

We show that $(\mathcal{X},\|\cdot\|)$ is a Banach space. Let $\left\{x_{n}\right\}$ be a Cauchy sequence in $\mathcal{X}$ and $\epsilon>0$ be given. Choose a natural number $N$ such that $\left\|x_{n}-x_{m}\right\|<\epsilon$ for all $m, n>N$. This implies that $\max _{t \in \mathbb{N}_{v-3}^{b+v+1}}\left|x_{n}(t)-x_{m}(t)\right|<\epsilon, \max _{t \in \mathbb{N}_{v-3}^{b+v+1}}\left|\Delta x_{n}(t)-\Delta x_{m}(t)\right|<\epsilon$ and

$$
\max _{t \in \mathbb{N}_{v-3}^{b+v+1}}\left|\Delta^{2} x_{n}(t)-\Delta^{2} x_{m}(t)\right|<\epsilon
$$

Choose $x(t), z(t), w(t) \in \mathbb{R}$ such that $x_{n}(t) \rightarrow x(t), \Delta x_{n}(t) \rightarrow z(t)$, and $\Delta^{2} x_{n}(t) \rightarrow w(t)$ for all $t \in \mathbb{N}_{v-3}^{b+v+1}$. Note that $\Delta x_{n}(t)=x_{n}(t+1)-x_{n}(t)$ and so $\Delta x(t)=x(t+1)-x(t)=z(t)$. Similarly, we get $\Delta^{2} x(t)=w(t)$. This implies that $\left|x_{n}(t)-x(t)\right|<\frac{\epsilon}{3},\left|\Delta x_{n}(t)-\Delta x(t)\right|<\frac{\epsilon}{3}$, and $\mid \Delta^{2} x_{n}(t)-$ $\Delta^{2} x(t) \mid<\frac{\epsilon}{3}$ for all $t \in \mathbb{N}_{\nu-3}^{b+v+1}$ and $n>M$ for some natural number $M$. Thus,

$$
\left\|x_{n}-x\right\|=\max _{t \in \mathbb{N}_{v-3}^{b+1}}\left|x_{n}(t)-x(t)\right|+\max _{t \in \mathbb{N}_{v-3}^{b+v+1}}\left|\Delta x_{n}(t)-\Delta x(t)\right|+\max _{t \in \mathbb{N}_{\nu-3}^{b+1+1}}\left|\Delta^{2} x(t)-\Delta^{2} x(t)\right|<\epsilon .
$$

Hence, $(\mathcal{X},\|\cdot\|)$ is a Banach space.

Let $x \in \mathcal{X}$. Define the set of selections of $F$ by

$$
S_{F, x}=\left\{y: \mathbb{N}_{0}^{b+1} \rightarrow \mathbb{R} \mid y(t) \in F\left(t, x(t), \Delta x(t), \Delta^{2} x(t)\right) \text { for all } t \in \mathbb{N}_{0}^{b+1}\right\} .
$$

Since $F\left(t, x(t), \Delta x(t), \Delta^{2} x(t)\right) \neq \emptyset$, the selection principle implies that $S_{F, x}$ is nonempty. 
Theorem 3.2 Suppose that $\psi \in \Psi$ and $F: \mathbb{N}_{v-3}^{b+v+1} \times \mathbb{R} \times \mathbb{R} \times \mathbb{R} \rightarrow P_{\mathrm{cp}}(\mathbb{R})$ is a multifunction such that

$$
H_{d}\left(F\left(t, x_{1}, x_{2}, x_{3}\right)-F\left(t, z_{1}, z_{2}, z_{3}\right)\right) \leq \psi\left(\left|x_{1}-z_{1}\right|+\left|x_{2}-z_{2}\right|+\left|x_{3}-z_{3}\right|\right)
$$

for all $t \in \mathbb{N}_{v-3}^{b+v+1}$ and $x_{1}, x_{2}, x_{3}, z_{1}, z_{2}, z_{3} \in \mathbb{R}$. Then the boundary value inclusion (3) has a solution.

Proof Choose $y \in S_{F, x}$ and put $h(t)=\sum_{s=0}^{b+1} G(t, s, \eta) y(s)$ for all $t \in \mathbb{N}_{v-3}^{v+b+1}$. Then $h \in \mathcal{X}$ and so the set

$$
\left\{h \in \mathcal{X} \text { : there exists } y \in S_{F, x} \text { such that } h(t)=\sum_{s=0}^{b+1} G(t, s, \eta) y(s) \text { for all } t \in \mathbb{N}_{v-3}^{b+v+1}\right\}
$$

is nonempty. Now define $\mathcal{F}: \mathcal{X} \rightarrow 2^{\mathcal{X}}$ by

$$
\begin{aligned}
\mathcal{F}(x)= & \left\{h \in \mathcal{X}: \text { there exists } y \in S_{F, x} \text { such that } h(t)=\sum_{s=0}^{b+1} G(t, s, \eta) y(s)\right. \\
& \text { for all } \left.t \in \mathbb{N}_{v-3}^{b+v+1}\right\} .
\end{aligned}
$$

We show that the multifunction $\mathcal{F}$ has a fixed point. First, we show that $\mathcal{F}(x)$ is closed subset of $\mathcal{X}$ for all $x \in \mathcal{X}$. Let $x \in \mathcal{X}$ and $\left\{u_{n}\right\}_{n \geq 1}$ be a sequence in $\mathcal{F}(x)$ with $u_{n} \rightarrow u$. For each $n$, choose $y_{n} \in S_{F, x}$ such that

$$
\begin{aligned}
& u_{n}(t)=\left[\frac{[\gamma+\delta(\nu-1)][(\eta+2-v)(\eta+3-v)] t^{\frac{\nu-3}{}}-\theta[\gamma+\delta(\nu-1)] t \frac{v-1}{}}{\theta \beta_{0} \mu \Gamma(\nu)(b+v) \underline{v-4}}\right. \\
& \left.-\frac{[\xi-\beta(v-3)][\gamma+\delta(v-1)][(\eta+2-v)(\eta+3-v)] t \underline{v-2}}{\beta(v-2) \theta \beta_{0} \mu \Gamma(v)(b+v) \underline{v-4}}\right] \\
& \times \sum_{s=0}^{b+1}(b-s+2)(b+v-\sigma(s))^{\frac{v-2}{}} y_{n}(s) \\
& +\left[\frac{\left[(\eta+2-v)(\eta+3-v)-\theta \beta_{0}\right] t \frac{v-3}{2}-\theta t \frac{v-1}{}}{\beta_{0} \theta^{2} \eta \frac{v-3}{\Gamma} \Gamma(v)}\right. \\
& \left.+\frac{[-\xi+\beta(v-3)]\left[(\eta+2-v)(\eta+3-v)-\theta \beta_{0}\right] t \frac{v-2}{-}}{\beta(v-2) \theta^{2} \beta_{0} \eta \frac{v-3}{} \Gamma(v)}\right] \\
& \times \sum_{s=0}^{\eta-v}(\eta-\sigma(s))^{\frac{\nu-1}{}} y_{n}(s)+\sum_{s=0}^{t-v} \frac{(t-\sigma(s))^{\frac{\nu-1}{}}}{\Gamma(\nu)} y_{n}(s)
\end{aligned}
$$

for all $t \in \mathbb{N}_{v-3}^{b+v+1}$. Since $F$ has compact values, $\left\{y_{n}\right\}_{n \geq 1}$ has a subsequence which converges to some $y \in S_{F, x}$. We denote this subsequence again by $\left\{y_{n}\right\}_{n \geq 1}$. So

$$
\begin{aligned}
u_{n}(t) & \rightarrow u(t) \\
& =\left[\frac{[\gamma+\delta(v-1)][(\eta+2-v)(\eta+3-v)] t \frac{v-3}{v}-\theta[\gamma+\delta(v-1)] t \frac{v-1}{\underline{v}}}{\theta \beta_{0} \mu \Gamma(v)(b+v)}\right.
\end{aligned}
$$




$$
\begin{aligned}
& \left.-\frac{[\xi-\beta(v-3)][\gamma+\delta(v-1)][(\eta+2-v)(\eta+3-v)] t^{\frac{\nu-2}{u}}}{\beta(v-2) \theta \beta_{0} \mu \Gamma(v)(b+v) \underline{v-4}}\right] \\
& \times \sum_{s=0}^{b+1}(b-s+2)(b+v-\sigma(s))^{\frac{v-2}{}} y(s) \\
& +\left[\frac{\left[(\eta+2-v)(\eta+3-v)-\theta \beta_{0}\right] t \underline{\nu-3}-\theta t^{\underline{\nu-1}}}{\beta_{0} \theta^{2} \eta \underline{v-3} \Gamma(v)}\right. \\
& \left.+\frac{[-\xi+\beta(\nu-3)]\left[(\eta+2-v)(\eta+3-v)-\theta \beta_{0}\right] t^{\nu-2}}{\beta(\nu-2) \theta^{2} \beta_{0} \eta \frac{v-3}{\underline{ }} \Gamma(v)}\right] \\
& \times \sum_{s=0}^{\eta-v}(\eta-\sigma(s))^{\frac{\nu-1}{-}} y(s)+\sum_{s=0}^{t-v} \frac{(t-\sigma(s))^{\frac{\nu-1}{v}}}{\Gamma(v)} y(s)
\end{aligned}
$$

for all $t \in \mathbb{N}_{v-3}^{b+v+1}$. This implies that $u \in \mathcal{F}(x)$. Thus, the multifunction $\mathcal{F}$ has closed values. Since $F$ is a compact multifunction, it is easy to check that $\mathcal{F}(x)$ is bounded set in $\mathcal{X}$ for all $x \in \mathcal{X}$. Let $x, z \in \mathcal{X}, h_{1} \in \mathcal{F}(x)$, and $h_{2} \in \mathcal{F}(z)$. Choose $y_{1} \in S_{F, x}$ and $y_{2} \in S_{F, z}$ such that

$$
\begin{aligned}
& h_{1}(t)=\left[\frac{[\gamma+\delta(\nu-1)][(\eta+2-v)(\eta+3-v)] t^{\nu-3}-\theta[\gamma+\delta(\nu-1)] t^{\underline{\nu-1}}}{\theta \beta_{0} \mu \Gamma(v)(b+v) \underline{\underline{v-4}}}\right. \\
& \left.-\frac{[\xi-\beta(v-3)][\gamma+\delta(v-1)][(\eta+2-v)(\eta+3-v)] t^{\underline{v-2}}}{\beta(v-2) \theta \beta_{0} \mu \Gamma(v)(b+v) \underline{v-4}}\right] \\
& \times \sum_{s=0}^{b+1}(b-s+2)(b+v-\sigma(s))^{\frac{\nu-2}{}} y_{1}(s) \\
& +\left[\frac{\left[(\eta+2-v)(\eta+3-v)-\theta \beta_{0}\right] t^{\nu-3}-\theta t \frac{\nu-1}{}}{\beta_{0} \theta^{2} \eta \frac{v-3}{\underline{v}} \Gamma(\nu)}\right. \\
& \left.+\frac{[-\xi+\beta(v-3)]\left[(\eta+2-v)(\eta+3-v)-\theta \beta_{0}\right] t \frac{v-2}{\underline{v}}}{\beta(v-2) \theta^{2} \beta_{0} \eta \frac{v-3}{\underline{ }} \Gamma(v)}\right] \\
& \times \sum_{s=0}^{\eta-v}(\eta-\sigma(s))^{\frac{v-1}{}} y_{1}(s)+\sum_{s=0}^{t-v} \frac{(t-\sigma(s)) \frac{\nu-1}{\Gamma}}{\Gamma(v)} y_{1}(s)
\end{aligned}
$$

and

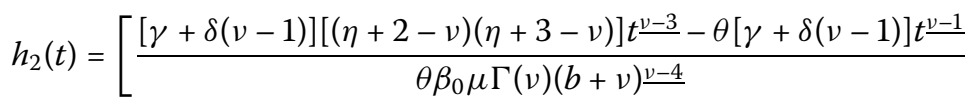

$$
\begin{aligned}
& \left.-\frac{[\xi-\beta(v-3)][\gamma+\delta(v-1)][(\eta+2-v)(\eta+3-v)] t^{\frac{\nu-2}{2}}}{\beta(v-2) \theta \beta_{0} \mu \Gamma(v)(b+v) \underline{v-4}}\right] \\
& \times \sum_{s=0}^{b+1}(b-s+2)(b+v-\sigma(s))^{\frac{\nu-2}{2}} y_{2}(s) \\
& +\left[\frac{\left[(\eta+2-v)(\eta+3-v)-\theta \beta_{0}\right] t^{\frac{\nu-3}{-}}-\theta t \frac{v-1}{\underline{v a-3}}}{\beta_{0} \theta^{2} \eta(v)}\right. \\
& \left.+\frac{[-\xi+\beta(v-3)]\left[(\eta+2-v)(\eta+3-v)-\theta \beta_{0}\right] t \underline{v-2}}{\beta(v-2) \theta^{2} \beta_{0} \eta \underline{v-3} \Gamma(v)}\right] \\
& \times \sum_{s=0}^{\eta-v}(\eta-\sigma(s))^{\frac{v-1}{v}} y_{2}(s)+\sum_{s=0}^{t-v} \frac{(t-\sigma(s))^{\frac{v-1}{}}}{\Gamma(v)} y_{2}(s)
\end{aligned}
$$


for all $t \in \mathbb{N}_{\nu-3}^{b+v+1}$. Since

$$
\begin{aligned}
& H_{d}\left(F\left(t, x(t), \Delta x(t), \Delta^{2} x(t)\right)-F\left(t, z(t), \Delta z(t), \Delta^{2} z(t)\right)\right) \\
& \quad \leq \psi\left(|x(t)-z(t)|+|\Delta x(t)-\Delta z(t)|+\left|\Delta^{2} x(t)-\Delta^{2} z(t)\right|\right)
\end{aligned}
$$

for all $x, z \in \mathcal{X}$ and $t \in \mathbb{N}_{v-3}^{b+v+1}$, we get

$$
\left|y_{1}(t)-y_{2}(t)\right| \leq \psi\left(|x(t)-z(t)|+|\Delta x(t)-\Delta z(t)|+\left|\Delta^{2} x(t)-\Delta^{2} z(t)\right|\right) .
$$

Now, put

$$
\begin{aligned}
& G_{1}=\max _{t \in \mathbb{N}_{v-3}^{b+1+v}}\left\{\mid \frac{[\gamma+\delta(\nu-1)][(\eta+2-v)(\eta+3-v)] t^{\frac{\nu-3}{\underline{v}}}-\theta[\gamma+\delta(\nu-1)] t^{\nu-1}}{\theta \beta_{0} \mu \Gamma(v)(b+v) \underline{\underline{v-4}}}\right. \\
& -\frac{[\xi-\beta(v-3)][\gamma+\delta(v-1)][(\eta+2-v)(\eta+3-v)] t \frac{v-2}{\underline{v}}}{\beta(v-2) \theta \beta_{0} \mu \Gamma(v)(b+v) \underline{v-4}} \mid \\
& \times \sum_{s=0}^{b+1}(b-s+2)(b+v-\sigma(s))^{\frac{v-2}{}}+\mid \frac{\left[(\eta+2-v)(\eta+3-v)-\theta \beta_{0}\right] t^{\frac{\nu-3}{}}-\theta t^{\frac{\nu-1}{L}}}{\beta_{0} \theta^{2} \eta \frac{v-3}{} \Gamma(v)} \\
& +\frac{[-\xi+\beta(v-3)]\left[(\eta+2-v)(\eta+3-v)-\theta \beta_{0}\right] t^{\frac{\nu-2}{}}}{\beta(v-2) \theta^{2} \beta_{0} \eta \underline{v-3} \Gamma(v)} \mid \\
& \left.\times \sum_{s=0}^{\eta-v}(\eta-\sigma(s))^{\frac{v-1}{w}}+\sum_{s=0}^{t-v} \frac{(t-\sigma(s))^{\frac{v-1}{}}}{\Gamma(v)}\right\} \\
& G_{2}=\max _{t \in \mathbb{N}_{v-3}^{b+1+v}}\left\{\mid \frac{(\nu-3)[\gamma+\delta(\nu-1)][(\eta+2-v)(\eta+3-v)] t \underline{v-4}}{\theta \beta_{0} \mu \Gamma(v)(b+v) \underline{\underline{v-4}}}\right. \\
& -\frac{(\nu-1) \theta[\gamma+\delta(v-1)] t^{\nu-2}}{\theta \beta_{0} \mu \Gamma(v)(b+v) \underline{\underline{\nu}-4}} \\
& -\frac{[\xi-\beta(v-3)][\gamma+\delta(v-1)][(\eta+2-v)(\eta+3-v)] t \frac{v-3}{\underline{v}}}{\beta \theta \beta_{0} \mu \Gamma(v)(b+v) \underline{v-4}} \\
& \times \sum_{s=0}^{b+1}(b-s+2)(b+v-\sigma(s))^{\underline{v-2}}
\end{aligned}
$$

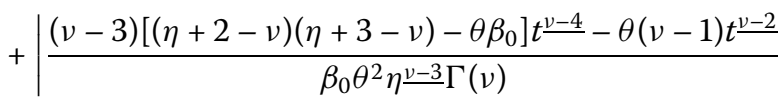

$$
\begin{aligned}
& +\frac{[-\xi+\beta(v-3)]\left[(\eta+2-v)(\eta+3-v)-\theta \beta_{0}\right] t \frac{v-3}{\underline{n}}}{\beta \theta^{2} \beta_{0} \eta \underline{\underline{v-3}} \Gamma(v)} \mid \\
& \left.\times \sum_{s=0}^{\eta-v}(\eta-\sigma(s))^{\frac{v-1}{}}+\sum_{s=0}^{t-v+1} \frac{(t-\sigma(s))^{\frac{\nu-2}{}}}{\Gamma(v-1)}\right\}
\end{aligned}
$$

and

$$
\begin{aligned}
G_{3}= & \max _{t \in \mathbb{N}_{v-3}^{b+1+v}}\left\{\mid \frac{(v-3)(v-4)[\gamma+\delta(v-1)][(\eta+2-v)(\eta+3-v)] t^{\frac{\nu-5}{}}}{\theta \beta_{0} \mu \Gamma(v)(b+v) \underline{v-4}}\right. \\
& -\frac{(\nu-1)(v-2) \theta[\gamma+\delta(v-1)] t^{\nu-3}}{\theta \beta_{0} \mu \Gamma(v)(b+v) \underline{v-4}}
\end{aligned}
$$




$$
\begin{aligned}
& -\frac{(\nu-3)[\xi-\beta(v-3)][\gamma+\delta(\nu-1)][(\eta+2-v)(\eta+3-v)] t^{\underline{v-4}}}{\beta \theta \beta_{0} \mu \Gamma(v)(b+v) \underline{v-4}} \mid \\
& \times \sum_{s=0}^{b+1}(b-s+2)(b+v-\sigma(s))^{\underline{v-2}} \\
& +\mid \frac{(v-3)(v-4)\left[(\eta+2-v)(\eta+3-v)-\theta \beta_{0}\right] t \frac{v-5}{-}-\theta(v-1)(v-2) t \frac{v-3}{-}}{\beta_{0} \theta^{2} \eta \underline{v-3} \Gamma(v)} \\
& +\frac{(v-3)[-\xi+\beta(v-3)]\left[(\eta+2-v)(\eta+3-v)-\theta \beta_{0}\right] t \frac{v-4}{}}{\beta \theta^{2} \beta_{0} \eta \frac{v-3}{\nu} \Gamma(v)} \mid \\
& \times \sum_{s=0}^{\eta-v}(\eta-\sigma(s))^{\frac{v-1}{v}}+\sum_{s=0}^{t-v+2} \frac{(t-\sigma(s)) \frac{v-3}{\Gamma(v-2)}}{\Gamma .}
\end{aligned}
$$

Then we have

$$
\begin{aligned}
& \left|h_{1}(t)-h_{2}(t)\right| \\
& =\mid\left[\frac{[\gamma+\delta(v-1)][(\eta+2-v)(\eta+3-v)] t^{\frac{\nu-3}{u}}-\theta[\gamma+\delta(\nu-1)] t^{\frac{\nu-1}{}}}{\theta \beta_{0} \mu \Gamma(v)(b+v) \underline{\underline{\nu}-4}}\right. \\
& \left.-\frac{[\xi-\beta(v-3)][\gamma+\delta(v-1)][(\eta+2-v)(\eta+3-v)] t \frac{v-2}{\underline{v}}}{\beta(v-2) \theta \beta_{0} \mu \Gamma(v)(b+v) \underline{v-4}}\right] \\
& \times \sum_{s=0}^{b+1}(b-s+2)(b+v-\sigma(s))^{\frac{v-2}{(}\left(y_{1}-y_{2}\right)(s)} \\
& +\left[\frac{\left[(\eta+2-v)(\eta+3-v)-\theta \beta_{0}\right] t^{\frac{v-3}{u}}-\theta t^{\frac{v-1}{u}}}{\beta_{0} \theta^{2} \eta \frac{v-3}{} \Gamma(v)}\right. \\
& \left.+\frac{[-\xi+\beta(v-3)]\left[(\eta+2-v)(\eta+3-v)-\theta \beta_{0}\right] t^{\frac{\nu-2}{2}}}{\beta(v-2) \theta^{2} \beta_{0} \eta \underline{v-3} \Gamma(v)}\right] \\
& \times \sum_{s=0}^{\eta-v}(\eta-\sigma(s))^{\frac{v-1}{}}\left(y_{1}-y_{2}\right)(s)+\sum_{s=0}^{t-v} \frac{(t-\sigma(s)) \frac{\nu-1}{\Gamma}}{\Gamma(v)}\left(y_{1}-y_{2}\right)(s) \mid \\
& \leq \mid \frac{[\gamma+\delta(\nu-1)][(\eta+2-v)(\eta+3-v)] t \frac{\nu-3}{\underline{v}}-\theta[\gamma+\delta(\nu-1)] t^{\underline{\nu-1}}}{\theta \beta_{0} \mu \Gamma(v)(b+v) \underline{\underline{v}}} \\
& -\frac{[\xi-\beta(\nu-3)][\gamma+\delta(v-1)][(\eta+2-v)(\eta+3-v)] t^{\frac{\nu-2}{}}}{\beta(v-2) \theta \beta_{0} \mu \Gamma(v)(b+v) \underline{v-4}} \mid \\
& \times \sum_{s=0}^{b+1}(b-s+2)(b+v-\sigma(s))^{\frac{v-2}{}}\left|y_{1}(s)-y_{2}(s)\right| \\
& +\mid \frac{\left[(\eta+2-v)(\eta+3-v)-\theta \beta_{0}\right] t^{\nu-3}-\theta t^{\frac{\nu-1}{}}}{\beta_{0} \theta^{2} \eta^{\frac{\nu-3}{}} \Gamma(v)} \\
& +\frac{[-\xi+\beta(v-3)]\left[(\eta+2-v)(\eta+3-v)-\theta \beta_{0}\right] t \frac{v-2}{\underline{n}}}{\beta(v-2) \theta^{2} \beta_{0} \eta \underline{v-3} \Gamma(v)} \mid \\
& \times \sum_{s=0}^{\eta-v}(\eta-\sigma(s))^{\frac{\nu-1}{}}\left|y_{1}(s)-y_{2}(s)\right|+\sum_{s=0}^{t-v} \frac{(t-\sigma(s)) \frac{\nu-1}{\Gamma}}{\Gamma(\nu)}\left|y_{1}(s)-y_{2}(s)\right| \\
& \leq \max _{t \in \mathbb{N}_{0}^{b+1}}\left|y_{1}(t)-y_{2}(t)\right|
\end{aligned}
$$




$$
\begin{aligned}
& \times \max _{t \in \mathbb{N}_{v-3}^{b+1+\nu}}\left\{\mid \frac{[\gamma+\delta(\nu-1)][(\eta+2-v)(\eta+3-v)] t^{\underline{\nu-3}}-\theta[\gamma+\delta(\nu-1)] t^{\nu-1}}{\theta \beta_{0} \mu \Gamma(\nu)(b+\nu) \underline{\nu-4}}\right. \\
& -\frac{[\xi-\beta(\nu-3)][\gamma+\delta(v-1)][(\eta+2-v)(\eta+3-v)] t^{\frac{\nu-2}{}}}{\beta(v-2) \theta \beta_{0} \mu \Gamma(v)(b+v) \underline{v-4}} \mid \\
& \times \sum_{s=0}^{b+1}(b-s+2)(b+v-\sigma(s))^{\frac{v-2}{}}+\mid \frac{\left[(\eta+2-v)(\eta+3-v)-\theta \beta_{0}\right] t^{\nu-3}-\theta t^{\nu-1}}{\beta_{0} \theta^{2} \eta \frac{v-3}{} \Gamma(v)} \\
& +\frac{[-\xi+\beta(\nu-3)]\left[(\eta+2-v)(\eta+3-v)-\theta \beta_{0}\right] t \frac{v-2}{2}}{\beta(v-2) \theta^{2} \beta_{0} \eta \underline{\underline{\nu-3}} \Gamma(v)} \mid \\
& \left.\times \sum_{s=0}^{\eta-v}(\eta-\sigma(s))^{\frac{\nu-1}{w}}+\sum_{s=0}^{t-v} \frac{(t-\sigma(s))^{\frac{\nu-1}{}}}{\Gamma(v)}\right\} \\
& \leq \psi\left(|x(t)-z(t)|+|\Delta x(t)-\Delta z(t)|+\left|\Delta^{2} x(t)-\Delta^{2} z(t)\right|\right) \times G_{1} .
\end{aligned}
$$

Since

$$
\begin{aligned}
& \Delta h_{1}(t)=\left[\frac{(\nu-3)[\gamma+\delta(v-1)][(\eta+2-v)(\eta+3-v)] t \frac{v-4}{n-}-(v-1) \theta[\gamma+\delta(v-1)] t \underline{v-2}}{\theta \beta_{0} \mu \Gamma(v)(b+v) \underline{v-4}}\right. \\
& \left.-\frac{[\xi-\beta(\nu-3)][\gamma+\delta(v-1)][(\eta+2-v)(\eta+3-v)] t \underline{v-3}}{\beta \theta \beta_{0} \mu \Gamma(v)(b+v) \underline{v-4}}\right] \\
& \times \sum_{s=0}^{b+1}(b-s+2)(b+v-\sigma(s))^{\frac{v-2}{}} y_{1}(s) \\
& +\left[\frac{(\nu-3)\left[(\eta+2-v)(\eta+3-v)-\theta \beta_{0}\right] t^{\frac{\nu-4}{}}-\theta(v-1) t \frac{v-2}{}}{\beta_{0} \theta^{2} \eta \frac{v-3}{} \Gamma(\nu)}\right. \\
& \left.+\frac{[-\xi+\beta(v-3)]\left[(\eta+2-v)(\eta+3-v)-\theta \beta_{0}\right] t \frac{v-3}{2}}{\beta \theta^{2} \beta_{0} \eta \frac{v-3}{-} \Gamma(v)}\right] \\
& \times \sum_{s=0}^{\eta-v}(\eta-\sigma(s))^{\frac{v-1}{}} y_{1}(s)+\sum_{s=0}^{t-v+1} \frac{(t-\sigma(s))^{\frac{v-2}{}}}{\Gamma(v-1)} y_{1}(s),
\end{aligned}
$$

we get

$$
\begin{aligned}
& \left|\Delta h_{1}(t)-\Delta h_{2}(t)\right| \\
& \leq \mid \frac{(v-3)[\gamma+\delta(v-1)][(\eta+2-v)(\eta+3-v)] t \frac{v-4}{n-4}-(v-1) \theta[\gamma+\delta(v-1)] t \frac{v-2}{2}}{\theta \beta_{0} \mu \Gamma(v)(b+v) \underline{v-4}} \\
& -\frac{[\xi-\beta(v-3)][\gamma+\delta(v-1)][(\eta+2-v)(\eta+3-v)] t \underline{v-3}}{\beta \theta \beta_{0} \mu \Gamma(v)(b+v) \underline{v-4}} \mid \\
& \times \sum_{s=0}^{b+1}(b-s+2)(b+v-\sigma(s))^{\frac{v-2}{}}\left|y_{1}(s)-y_{2}(s)\right| \\
& +\mid \frac{(v-3)\left[(\eta+2-v)(\eta+3-v)-\theta \beta_{0}\right] t^{\frac{\nu-4}{}}-\theta(v-1) t^{\underline{\nu-2}}}{\beta_{0} \theta^{2} \eta \frac{v-3}{\underline{ }} \Gamma(v)} \\
& +\frac{[-\xi+\beta(\nu-3)]\left[(\eta+2-v)(\eta+3-v)-\theta \beta_{0}\right] t \frac{v-3}{}}{\beta \theta^{2} \beta_{0} \eta \frac{v-3}{L} \Gamma(\nu)} \mid \\
& \times \sum_{s=0}^{\eta-v}(\eta-\sigma(s))^{\frac{v-1}{}}\left|y_{1}(s)-y_{2}(s)\right|+\sum_{s=0}^{t-v+1} \frac{(t-\sigma(s))^{\frac{v-2}{2}}}{\Gamma(v-1)}\left|y_{1}(s)-y_{2}(s)\right|
\end{aligned}
$$




$$
\begin{aligned}
& \leq \max _{t \in \mathbb{N}_{0}^{b+1}}\left|y_{1}(t)-y_{2}(t)\right| \\
& \times \max _{t \in \mathbb{N}_{v-3}^{b+1+\nu}}\left\{\mid \frac{(\nu-3)[\gamma+\delta(\nu-1)][(\eta+2-v)(\eta+3-v)] t^{\frac{\nu-4}{\underline{y}}}}{\theta \beta_{0} \mu \Gamma(v)(b+v) \underline{\underline{\nu-4}}}\right. \\
& -\frac{(v-1) \theta[\gamma+\delta(v-1)] t \frac{v-2}{\underline{v}}}{\theta \beta_{0} \mu \Gamma(v)(b+v) \underline{\underline{v-4}}} \\
& -\frac{[\xi-\beta(\nu-3)][\gamma+\delta(v-1)][(\eta+2-v)(\eta+3-v)] t^{\frac{\nu-3}{}}}{\beta \theta \beta_{0} \mu \Gamma(v)(b+v) \underline{v-4}} \mid \\
& \times \sum_{s=0}^{b+1}(b-s+2)(b+v-\sigma(s))^{\underline{v-2}} \\
& +\mid \frac{(v-3)\left[(\eta+2-v)(\eta+3-v)-\theta \beta_{0}\right] t \frac{v-4}{\underline{v}}-\theta(v-1) t \underline{v-2}}{\beta_{0} \theta^{2} \eta^{\underline{\nu-3}} \Gamma(v)} \\
& +\frac{[-\xi+\beta(\nu-3)]\left[(\eta+2-v)(\eta+3-v)-\theta \beta_{0}\right] t^{\nu-3}}{\beta \theta^{2} \beta_{0} \eta \frac{\nu-3}{\underline{ }} \Gamma(v)} \mid \\
& \left.\times \sum_{s=0}^{\eta-v}(\eta-\sigma(s))^{\frac{\nu-1}{t}}+\sum_{s=0}^{t-v+1} \frac{(t-\sigma(s))^{\frac{\nu-2}{}}}{\Gamma(v-1)}\right\} \\
& \leq \psi\left(|x(t)-z(t)|+|\Delta x(t)-\Delta z(t)|+\left|\Delta^{2} x(t)-\Delta^{2} z(t)\right|\right) \times G_{2} .
\end{aligned}
$$

Also, we have

$$
\begin{aligned}
& \left|\Delta^{2} h_{1}(t)-\Delta^{2} h_{2}(t)\right| \\
& \leq \mid \frac{(v-3)(v-4)[\gamma+\delta(v-1)][(\eta+2-v)(\eta+3-v)] t^{\underline{\nu-5}}}{\theta \beta_{0} \mu \Gamma(v)(b+v) \underline{\underline{v-4}}} \\
& -\frac{(v-1)(v-2) \theta[\gamma+\delta(v-1)] t \underline{v-3}}{\theta \beta_{0} \mu \Gamma(v)(b+v) \underline{v-4}} \\
& -\frac{(\nu-3)[\xi-\beta(v-3)][\gamma+\delta(v-1)][(\eta+2-v)(\eta+3-v)] t^{\underline{\nu-4}}}{\beta \theta \beta_{0} \mu \Gamma(v)(b+v) \underline{\underline{v-4}}} \mid \\
& \times \sum_{s=0}^{b+1}(b-s+2)(b+v-\sigma(s))^{\frac{v-2}{}}\left|y_{1}(s)-y_{2}(s)\right| \\
& +\mid \frac{(v-3)(v-4)\left[(\eta+2-v)(\eta+3-v)-\theta \beta_{0}\right] t^{\frac{\nu-5}{}}-\theta(v-1)(v-2) t \frac{v-3}{}}{\beta_{0} \theta^{2} \eta \frac{v-3}{} \Gamma(v)} \\
& +\frac{(\nu-3)[-\xi+\beta(\nu-3)]\left[(\eta+2-v)(\eta+3-v)-\theta \beta_{0}\right] t^{\frac{\nu-4}{u}}}{\beta \theta^{2} \beta_{0} \eta \frac{v-3}{} \Gamma(v)} \mid \\
& \times \sum_{s=0}^{\eta-v}(\eta-\sigma(s))^{\frac{\nu-1}{}}\left|y_{1}(s)-y_{2}(s)\right|+\sum_{s=0}^{t-v+2} \frac{(t-\sigma(s))^{\frac{\nu-3}{}}}{\Gamma(\nu-2)}\left|y_{1}(s)-y_{2}(s)\right| \\
& \leq \max _{t \in \mathbb{N}_{0}^{b+1}}\left|y_{1}(t)-y_{2}(t)\right| \\
& \times \max _{t \in \mathbb{N}_{\nu-3}^{b+1+\nu}}\left\{\mid \frac{(v-3)(v-4)[\gamma+\delta(v-1)][(\eta+2-v)(\eta+3-v)] t \frac{v-5}{\underline{v}}}{\theta \beta_{0} \mu \Gamma(v)(b+v)^{\underline{\nu-4}}}\right. \\
& -\frac{(v-1)(v-2) \theta[\gamma+\delta(v-1)] t \underline{v-3}}{\theta \beta_{0} \mu \Gamma(v)(b+v) \underline{v-4}}
\end{aligned}
$$




$$
\begin{aligned}
& -\frac{(v-3)[\xi-\beta(v-3)][\gamma+\delta(v-1)][(\eta+2-v)(\eta+3-v)] t^{\underline{\nu-4}}}{\beta \theta \beta_{0} \mu \Gamma(v)(b+v) \underline{v-4}} \mid \\
& \times \sum_{s=0}^{b+1}(b-s+2)(b+v-\sigma(s))^{\underline{v-2}} \\
& +\mid \frac{(v-3)(v-4)\left[(\eta+2-v)(\eta+3-v)-\theta \beta_{0}\right] t^{\nu-5}-\theta(v-1)(v-2) t \frac{v-3}{}}{\beta_{0} \theta^{2} \eta \frac{v-3}{} \Gamma(v)} \\
& +\frac{(\nu-3)[-\xi+\beta(\nu-3)]\left[(\eta+2-v)(\eta+3-v)-\theta \beta_{0}\right] t^{\nu-4}}{\beta \theta^{2} \beta_{0} \eta \frac{v-3}{\underline{v}} \Gamma(v)} \mid \\
& \left.\times \sum_{s=0}^{\eta-v}(\eta-\sigma(s))^{\frac{\nu-1}{v}}+\sum_{s=0}^{t-v+2} \frac{(t-\sigma(s))^{\frac{\nu-3}{}}}{\Gamma(\nu-2)}\right\} \\
& \leq \psi\left(|x(t)-z(t)|+|\Delta x(t)-\Delta z(t)|+\left|\Delta^{2} x(t)-\Delta^{2} z(t)\right|\right) \times G_{3} .
\end{aligned}
$$

Hence, we obtain

$$
\begin{aligned}
\left\|h_{1}-h_{2}\right\|= & \max _{t \in \mathbb{N}_{v-3}^{b+1+\nu}}\left|h_{1}(t)-h_{2}(t)\right|+\max _{t \in \mathbb{N}_{v-3}^{b+1+\nu}}\left|\Delta h_{1}(t)-\Delta h_{2}(t)\right| \\
& +\max _{t \in \mathbb{N}_{v-3}^{b+1+\nu}}\left|\Delta^{2} h_{1}(t)-\Delta^{2} h_{2}(t)\right| \\
\leq & \psi\left(|x(t)-z(t)|+|\Delta x(t)-\Delta z(t)|+\left|\Delta^{2} x(t)-\Delta^{2} z(t)\right|\right)\left(G_{1}+G_{2}+G_{3}\right) \\
\leq & \left(G_{1}+G_{2}+G_{3}\right) \psi(\|x-z\|)
\end{aligned}
$$

for all $x, z \in \mathcal{X}, h_{1} \in \mathcal{F}(x)$, and $h_{2} \in \mathcal{F}(z)$. So $H_{d}(\mathcal{F}(x), \mathcal{F}(z)) \leq\left(G_{1}+G_{2}+G_{3}\right) \psi(\|x-z\|)$ for all $x, z \in \mathcal{X}$.

Define the function $\alpha$ on $\mathcal{X} \times \mathcal{X}$ by $\alpha(x, z)=1$ whenever $G_{1}+G_{2}+G_{3}<1$ and $\alpha(x, z)=$ $\frac{1}{G_{1}+G_{2}+G_{3}}$ otherwise. Thus,

$$
\alpha(x, z) H_{d}(\mathcal{F}(x), \mathcal{F}(z)) \leq \psi(\|x-z\|)
$$

for all $x, z \in \mathcal{X}$. Let $\left\{x_{n}\right\}$ be a sequence in $\mathcal{X}$ with $\alpha\left(x_{n}, x_{n+1}\right) \geq 1$ for all $n$ and $x_{n} \rightarrow x$. Then it is easy to check that there exists a subsequence $\left\{x_{n_{k}}\right\}$ of $\left\{x_{n}\right\}$ such that $\alpha\left(x_{n_{k}}, x\right) \geq 1$ for all $k$. This implies that $\mathcal{X}$ obeys the condition $\left(C_{\alpha}\right)$. If $x \in \mathcal{X}$ and $y \in \mathcal{F}(x)$ with $\alpha(x, y) \geq 1$, then it is easy to see that $\alpha(y, z) \geq 1$ for all $z \in \mathcal{F}(y)$. Thus, $\mathcal{F}$ is an $\alpha$-admissible $\alpha$ - $\psi$-contractive multifunction. Hence by using Theorem 2.2, there exists $x^{*} \in \mathcal{X}$ such that $x^{*} \in \mathcal{F}\left(x^{*}\right)$. One can check that $x^{*}$ is a solution for the problem (3).

Example 3.1 Consider the fractional finite difference inclusion

$$
\Delta_{-0.5}^{2.5} x(t) \in\left[1, e^{t^{2}}+2+\frac{\sin x(t)}{e^{2|t|}}+\sinh ^{2} t+\frac{|\Delta x(t)|}{4|t|}+\frac{3}{6 t^{2}-1}+\frac{\left|\Delta^{2} x(t)\right|}{\cosh |3 t|}\right]
$$

via the boundary value conditions $\xi x(-0.5)+\beta \Delta x(-0.5)=0, \gamma x(6.5)+\delta \Delta x(6.5)=0$, and $x(3.5)=0$, where $\xi, \beta, \gamma, \delta$ are non-zero numbers. In fact, this problem is a special case of the problem (3), where $v=2.5, \eta=3.5, b=4$, and

$$
F\left(t, x_{1}, x_{2}, x_{3}\right)=\left[1, e^{t^{2}}+2+\frac{\sin x_{1}}{e^{2|t|}}+\sinh ^{2} t+\frac{\left|x_{2}\right|}{4|t|}+\frac{3}{6 t^{2}-1}+\frac{\left|x_{3}\right|}{\cosh |3 t|}\right] .
$$


Note that $e^{t^{2}}+2+\frac{\sin x_{1}}{e^{2|t|}}+\sinh ^{2} t+\frac{\left|x_{2}\right|}{4|t|}+\frac{3}{6 t^{2}-1}+\frac{\left|x_{3}\right|}{\cosh |3 t|}>1$ for all $t \in \mathbb{N}_{-0.5}^{7.5}$ and $x_{1}, x_{2}, x_{3} \in \mathbb{R}$. Also, $e^{2|t|} \geq 2,4|t| \geq 2$, and $\cosh |3 t| \geq 2$ for all $t \in \mathbb{N}_{-0.5}^{7.5}$ and $F$ is a compact valued multifunction on $\mathbb{N}_{-0.5}^{7.5} \times \mathbb{R} \times \mathbb{R} \times \mathbb{R}$. Now, define $\psi \in \Psi$ by $\psi(z)=\frac{z}{2}$ for all $z \geq 0$. Since

$$
\begin{aligned}
& H_{d}\left(F\left(t, x_{1}, x_{2}, x_{3}\right), F\left(t, z_{1}, z_{2}, z_{3}\right)\right) \\
& \quad \leq \mid \frac{\sin x_{1}}{e^{2|t|}}-\frac{x_{2}}{4|t|}+\frac{x_{3}}{\cosh |3 t|}-\frac{\sin z_{1}}{e^{2|t|}+\frac{z_{2}}{4|t|}-\frac{z_{3}}{\cosh |3 t|} \mid} \\
& \quad \leq \frac{\left|x_{1}-z_{1}\right|+\left|x_{2}-z_{2}\right|+\left|x_{3}-z_{3}\right|}{2} \\
& \quad=\psi\left(\left|x_{1}-z_{1}\right|+\left|x_{2}-z_{2}\right|+\left|x_{3}-z_{3}\right|\right)
\end{aligned}
$$

for all $t \in \mathbb{N}_{-0.5}^{7.5}$ and $x_{1}, x_{2}, x_{3}, z_{1}, z_{2}, z_{3} \in \mathbb{R}$, by using Theorem 3.2 the problem (4) has at least one solution.

\section{Conclusions}

In this manuscript, based on a fixed point theorem, we provided the existence result for a fractional finite difference inclusion in the presence of the general boundary conditions. An example illustrates our result.

\section{Competing interests}

The authors declare that they have no competing interests.

\section{Authors' contributions}

All authors contributed equally to the writing of this paper. All authors read and approved the final manuscript.

\section{Author details}

'Department of Mathematics, Cankaya University, Ogretmenler Cad. 14, Balgat, Ankara, 06530, Turkey. ${ }^{2}$ Institute of Space Sciences, Magurele, Bucharest, Romania. ${ }^{3}$ Department of Mathematics, Azarbaijan Shahid Madani University, Tabriz, Iran.

\section{Acknowledgements}

The research of the second and third authors was supported by Azarbaijan Shahid Madani University.

Received: 14 April 2015 Accepted: 30 June 2015 Published online: 06 August 2015

\section{References}

1. Awasthi, P: Boundary value problems for discrete fractional equations. PhD thesis, University of Nebraska-Lincoln, Ann Arbor, Ml (2013)

2. Baleanu, D: A k-dimensional system of fractional finite difference equations. Abstr. Appl. Anal. 2014, Article ID 312578 (2014)

3. Goodrich, CS: On a fractional boundary value problem with fractional boundary conditions. Appl. Math. Lett. 25, 1101-1105 (2012)

4. Goodrich, CS: On discrete sequential fractional boundary value problems. J. Math. Anal. Appl. 385, 111-124 (2012)

5. Goodrich, CS: Solutions to a discrete right-focal fractional boundary value problem. Int. J. Differ. Equ. 5, 195-216 (2010)

6. Goodrich, CS: Some new existence results for fractional difference equations. Int. J. Dyn. Syst. Differ. Equ. 3, 145-162 (2011)

7. Pan, Y, Han, Z, Sun, S, Zhao, Y: The existence of solutions to a system of discrete fractional boundary value problems. Abstr. Appl. Anal. 2012, Article ID 707631 (2012)

8. Acar, N, Atici, FM: Exponential functions of discrete fractional calculus. Appl. Anal. Discrete Math. 7, 343-353 (2013)

9. Atici, FM, Sengul, S: Modeling with fractional difference equations. J. Math. Anal. Appl. 369, 1-9 (2010)

10. Atici, FM, Eloe, PW: Initial value problems in discrete fractional calculus. Proc. Am. Math. Soc. 137, 981-989 (2009)

11. Elaydi, SN: An Introduction to Difference Equations. Springer, Berlin (1996)

12. Holm, M: Sum and differences compositions in discrete fractional calculus. CUBO 13, 153-184 (2011)

13. Holm, M: The theory of discrete fractional calculus: development and applications. PhD thesis, University of Nebraska-Lincoln, Ann Arbor, MI (2011)

14. Kang, $S, L i, Y, C$ Chen, $H$ : Positive solutions to boundary value problems of fractional difference equation with nonlocal conditions. Adv. Differ. Equ. 2014, 7 (2014)

15. Mohan, JJ, Deekshitulu, GVSR: Fractional order difference equations. Int. J. Differ. Equ. 2012, Article ID 780619 (2012)

16. Agarwal, RP, Ahmad, B: Existence theory for anti-periodic boundary value problems of fractional differential equations and inclusions. J. Appl. Math. Comput. 62, 1200-1214 (2011) 
17. Agarwal, RP, Belmekki, M, Benchohra, M: A survey on semilinear differential equations and inclusions involving Riemann-Liouville fractional derivative. Adv. Differ. Equ. 2009, Article ID 981728 (2009)

18. Agarwal, RP, Benchohra, M, Hamani, S: A survey on existence results for boundary value problems of nonlinear fractional differential equations and inclusions. Acta Appl. Math. 109, 973-1033 (2010)

19. Ahmad, B, Nieto, JJ: Existence of solutions for anti-periodic boundary value problems involving fractional differential equations via Leray-Schauder degree theory. Topol. Methods Nonlinear Anal. 35, 295-304 (2010)

20. Ahmad, B, Ntouyas, SK: Boundary value problem for fractional differential inclusions with four-point integral boundary conditions. Surv. Math. Appl. 6, 175-193 (2011)

21. Aubin, J, Ceuina, A: Differential Inclusions: Set-Valued Maps and Viability Theory. Springer, Berlin (1984)

22. El-Sayed, AMA, Ibrahim, AG: Multivalued fractional differential equations. Appl. Math. Comput. 68, 15-25 (1995)

23. Kisielewicz, M: Differential Inclusions and Optimal Control. Kluwer Academic, Dordrecht (1991)

24. Liu, X, Liu, Z: Existence result for fractional differential inclusions with multivalued term depending on lower-order derivative. Abstr. Appl. Anal. 2012, Article ID 423796 (2012)

25. Goodrich, CS: A comparison result for the fractional difference operator. Int. J. Differ. Equ. 6, 17-37 (2011)

26. Mohammadi, B, Rezapour, S, Shahzad, N: Some results on fixed points of $\xi$ - $\psi$-Ciric generalized multifunctions. Fixed Point Theory Appl. 2013, 24 (2013)

\section{Submit your manuscript to a SpringerOpen ${ }^{\circ}$ journal and benefit from:}

- Convenient online submission

Rigorous peer review

- Immediate publication on acceptance

- Open access: articles freely available online

- High visibility within the field

- Retaining the copyright to your article 